PHYSICAL REVIEW D 94, 024049 (2016)

\title{
Initial directional singularity in inflationary models
}

\author{
L. Fernández-Jambrina* \\ Matemática Aplicada, E.T.S.I. Navales, Universidad Politécnica de Madrid, \\ Arco de la Victoria 4, E-28040 Madrid, Spain \\ (Received 23 June 2016; published 26 July 2016)
}

\begin{abstract}
In Haro, Amorós, and Pan [Phys. Rev. D 93, 084018 (2016)] a new cosmological model is proposed with no big bang singularity in the past, though past geodesically incomplete. This model starts with an inflationary era, follows with a stiff matter dominated period and evolves to accelerated expansion in an asymptotically de Sitter regime in a realistic fashion. The big bang singularity is replaced by a directional singularity. This singularity cannot be reached by comoving observers, since it would take them an infinite proper time lapse to go back to it. On the contrary, observers with nonzero linear momentum have the singularity at finite proper time in their past, though arbitrarily large. Hence, the time lapse from the initial singularity can be as long as desired, even infinity, depending on the linear momentum of the observer. This conclusion applies to similar inflationary models. Due to the interest of these models, we address here the properties of such singularities.
\end{abstract}

DOI: 10.1103/PhysRevD.94.024049

\section{INTRODUCTION}

The accelerated expansion of our Universe [1-5] has motivated the consideration of either new ingredients in the energy content of cosmological models [6-8] or corrections to the general theory of gravitation compatible with observations [9-12].

As a consequence, some energy conditions are violated by these new ingredients with the result of new future scenarios for our universe in the form of new singularities (big rip, sudden singularities...) or nonsingular asymptotic behaviors observationally undistinguishable from singularities (pseudorip, little rip...). Some of these singularities are weak in the sense that the universe can be extended beyond the singularity and in consequence it cannot be considered the end of the universe. These phenomena have also been discovered recently in inflationary models [13].

But these singular behaviors may also appear at the beginning of our universe, replacing the traditional big bang as initial singularity. One of these models is [14], but other inflationary models [15] follow a similar pattern.

The model [14] proposes a simple equation of state which succeeds in removing the big bang singularity, replacing it by another one, dubbed little bang in analogy with the little rip, and producing an inflationary era. A phase transition stops the inflation until in the far future accelerated expansion is dominant. An interesting feature shown in [14] is that the new singularity is at infinite cosmic time, for comoving observers, but at finite proper time for non-comoving observers. This resembles the behavior of directional singularities in $[16,17]$.

We would like to comment here the nature and properties of these initial singularities appearing in some inflationary

*leonardo.fernandez@upm.es; http://dcain.etsin.upm.es/ilfj.htm cosmological models. We begin by reviewing the possible singular scenarios in Sec. II, paying special attention to directional singularities in Sec. III in order to frame the inflationary model in Sec. IV. The derived conclusions are summarized in the final section.

\section{COSMOLOGICAL SINGULARITIES}

In [17] a thorough classification of cosmological singularities has been provided both at finite and infinite coordinate time, obtained in terms of either the behavior of the barotropic index $w$ for flat models of scale factor $a$ or equivalently the deceleration parameter $q$,

$$
w=\frac{p}{\rho}=-\frac{1}{3}-\frac{2}{3} \frac{a \ddot{a}}{\dot{a}^{2}}, \quad q=-\frac{a \ddot{a}}{\dot{a}^{2}}=\frac{1+3 w}{2},
$$

where $\rho$ is the energy density and $p$ is the pressure of the model and the dot stands for derivative with respect to coordinate time $t$.

This classification makes use of generalized power expansions [18] in coordinate time of the deviation $h$ from the pure cosmological constant case,

$$
w(t)=-1+\frac{2}{3} h(t), \quad q(t)=-1-h(t),
$$

and extends the one in [19], which has been enlarged in [20-22]. We shall not include here nonsingular behaviors such as little rip [23], pseudorip [24] and the little sibling of the big rip [25], since we are concerned just with singularities, though they are also taken into account in [17]. The classification can be summarized as follows:

(i) Type -1: "Grand bang/rip": [17] The scale factor vanishes or blows up at $w=-1$. The Hubble ratio, the energy density and the pressure blow up. These are strong singularities. 
(ii) Type 0: "Big bang": The scale factor vanishes at $w \neq-1$. The Hubble ratio, the energy density and the pressure blow up. These are also strong.

(iii) Type I: "Big rip" [26]: The scale factor, the Hubble ratio, the energy density and the pressure blow up. Null geodesics are complete, but not timelike geodesics. They are strong singularities.

(iv) Type II: "Sudden singularities" [27,28]: They have been also dubbed "quiescent singularities" [29]: The scale factor, the Hubble ratio and the energy density remain finite, whereas the pressure blows up. That is, the second derivative of the scale factor diverges. Some subcases have been dubbed big brake [30] and big boost [31]. These are weak singularities [32] and the models just violate the dominant energy condition.

(v) Type III: "Big freeze" [33] or "finite scale factor singularities": The scale factor remains finite, but the Hubble factor, the energy density and the pressure blow up. That is, the first derivative of the scale factor is singular. Depending on the definition used $[34,35]$, they can be either strong or weak [36].

(vi) Type IV [37]: The scale factor, the Hubble ratio, the energy density and the pressure are finite, whereas higher derivatives of the scale factor blow up. They are dubbed "generalized sudden singularities" if the barotropic index $w$ is finite [22] and big separation if it blows up with vanishing pressure and energy density. These are weak singularities.

(vii) Type V: " $w$-singularities" [38,39]: The scale factor, the Hubble ratio, the energy density, the pressure and higher derivatives of the scale factor are finite, whereas the barotropic index $w$ blows up. They are weak singularities [40].

(viii) Type $\infty$ : "Directional singularities" [16]: These type of singularities appear at infinite coordinate time, but at finite proper time, at least for some observers. In this sense they are directional. These are p.p. curvature singularities (curvature singularities along a parallelly transported basis) [41]. We pay little attention to this overlooked type of singularities.

This analysis has been done at the classical level. It must be taken into account that some of these singularities have been shown to be removable on considering quantum gravity [42] and loop quantum gravity corrections [43].

\section{TYPE $\infty$ SINGULARITIES}

Type $\infty$ singularities appear at coordinate time $t= \pm \infty$. In general, this time is inaccessible, but this is not so in certain cosmological models.

For a flat Friedmann-Lemaître-Robinson-Walker cosmological model with scale factor $a(t)$ and metric

$$
d s^{2}=-d t^{2}+a^{2}(t)\left(d r^{2}+r^{2}\left(d \theta^{2}+\sin ^{2} \theta d \phi^{2}\right)\right),
$$

we notice [16] that the system of equations for geodesic curves, followed by nonaccelerated observers $(\delta=1)$ and lightlike particles $(\delta=0)$ with specific linear momentum $P$, can be reduced to

$$
\begin{aligned}
& \frac{d t}{d \tau}=\sqrt{\delta+\frac{P^{2}}{a^{2}(t)},} \\
& \frac{d r}{d \tau}= \pm \frac{P}{a^{2}(t)},
\end{aligned}
$$

for constant $\theta$ and $\phi$, due to the symmetry of these models, and where $\tau$ is the intrinsic or proper time as measured by the observer.

For null geodesics we have

$$
\frac{d t}{d \tau}=\frac{P}{a(t)} \Rightarrow \Delta \tau=\frac{1}{P} \int_{-\infty}^{t} a(t) d t
$$

Hence, for the initial event $t=-\infty$ to be at a finite proper time lapse $\Delta \tau$ of an event at $t$, we require

$$
\int_{-\infty}^{t} a(t) d t<\infty
$$

That is, singular behavior at $t=-\infty$ only may appear if the scale factor is an integrable function of coordinate time. This means that it is necessary, but not sufficient, that $a(t)$ tends to zero when $t$ tends to $-\infty$.

Similarly, for timelike geodesics with nonzero $P$,

$$
\Delta \tau=\int_{-\infty}^{t} \frac{d t}{\sqrt{1+\frac{P^{2}}{a^{2}(t)}}}<\frac{1}{P} \int_{-\infty}^{t} a(t) d t,
$$

the proper time lapse to $t=-\infty$ is finite if the time lapse for lightlike geodesics is finite and then $t=-\infty$ is accessible for these observers.

Hence, condition (3) implies that both lightlike and timelike geodesics with nonzero $P$ have $t=-\infty$ at a finite proper time lapse in their past.

On the contrary, comoving observers, following timelike geodesics with $P=0$, have $d \tau=d t$ and therefore $t=-\infty$ is for them at an infinite proper time lapse in the past and cannot have experienced the singularity.

This is the reason why Type $\infty$ singularities are directional, in the sense that they are accessible for causal geodesics, except for those with $P=0$.

According to [17], Type $\infty$ singularities may appear in three instances:

(i) Finite $\int_{-\infty} h d t, \quad h(t)>0: \quad a_{-\infty}=0, \quad \rho_{-\infty}=\infty$, $p_{-\infty}=-\infty, w_{-\infty}=-1$. They differ from little rip in the sign of $h(t)$, so they can be dubbed little bang if it is an initial singularity or little crunch [17] if it is 
a final singularity. Instances of this case are models with scale factor $a(t) \propto e^{-\alpha(-t)^{p}}$ with $p>1, \alpha>0$.

(ii) $h_{-\infty}=0,|h(t)| \gtrsim|t|^{-1}, h(t)<0: a_{-\infty}=0, \rho_{-\infty}=0$, $p_{-\infty}=0, w_{-\infty}=-1$. By changing the sign of $h(t)$ we obtain a sort of little rip with vanishing asymptotic energy density and pressure. Examples for this case are models with scale factor $a(t) \propto e^{-\alpha(-t)^{p}}$ with $p \in(0,1), \alpha>0$.

(iii) Finite $h_{-\infty} \in(-1,0): a_{-\infty}=0, \rho_{-\infty}=0, p_{-\infty}=0$, finite $w_{-\infty} \neq-1$. This is the case, for instance, of models with $a(t) \propto t^{-p}, p>1$, as the ones studied in [16].

It is interesting to check the strength of these singularities in order to know if the model can be extended beyond the singularity.

There are several definitions of strong singularities. The concept comes up first in [44] by defining a strong curvature singularity as one for which no object "can arrive intact at the singularity".

Tipler [34] clarifies the concept by defining a strong curvature singularity as one for which "any object hitting it is crushed to zero volume." The volume of the object is rigorously defined by any three linearly independent spacelike vorticity-free Jacobi fields orthogonal to the velocity of the geodesic. This definition is equivalent to inextendibility of the spacetime in a continuous fashion beyond the singularity.

In the context of cosmic censorship Królak [35] proposed another definition which requires that, instead of a vanishing volume of the object, the derivative of the volume must be negative close to the singularity.

Such definitions are complex to apply from scratch, but fortunately there are necessary and sufficient conditions for their requirements [45]. They are even simpler in our case, since Friedmann-Lemaître-Robinson-Walker spacetimes are conformally flat.

For instance, according to Tipler, a null geodesic ends up at a strong singularity at proper time $\tau_{0}$ if and only if

$$
\int_{0}^{\tau} d \tau^{\prime} \int_{0}^{\tau^{\prime}} d \tau^{\prime \prime} R_{i j} u^{i} u^{j}
$$

blows up as $\tau$ tends to $\tau_{0} . R$ is the Ricci tensor of the spacetime and $u$ is the velocity of the geodesic.

According to Królak, a null geodesic ends up at a strong singularity at $\tau_{0}$ if and only if

$$
\int_{0}^{\tau} d \tau^{\prime} R_{i j} u^{i} u^{j}
$$

blows up as $\tau$ tends to $\tau_{0}$.

For timelike geodesics the previous conditions become just sufficient conditions.
Let us check these requirements for the first two subtypes of singularities. For the third subtype the strength was checked in [16].

For a null geodesic, the components of the velocity $u$ are

$$
u^{t}=\frac{d t}{d \tau}=\frac{P}{a}, \quad u^{r}=\frac{d r}{d \tau}= \pm \frac{P}{f a^{2}},
$$

and hence the Ricci curvature along the geodesic takes the expression

$$
\begin{aligned}
R_{i j} u^{i} u^{j} d \tau & =2 P^{2}\left(\frac{\dot{a}^{2}}{a^{4}}-\frac{\ddot{a}}{a^{3}}\right) d \tau \\
& =2 P\left(\frac{\dot{a}^{2}}{a^{3}}-\frac{\ddot{a}}{a^{4}}\right) d t \\
& =-2 P \ddot{x} e^{-x} d t,
\end{aligned}
$$

in terms of $x(t)=\ln a(t)$.

For timelike geodesics,

$$
u^{t}=\sqrt{1+\frac{P^{2}}{a^{2}}}, \quad u^{r}= \pm \frac{P}{a^{2}},
$$

since $a_{-\infty}=0$ we have

$$
\begin{aligned}
R_{i j} u^{i} u^{j} d \tau & =\frac{-\frac{3 \ddot{a}}{a}+2 P^{2}\left(\frac{\dot{a}^{2}}{a^{4}}-\frac{\ddot{a}}{a^{3}}\right)}{\sqrt{1+\frac{P^{2}}{a^{2}}}} d t \\
& \simeq\left(-\frac{3 \ddot{a}}{P}+2 P\left(\frac{\dot{a}^{2}}{a^{3}}-\frac{\ddot{a}}{a^{2}}\right)\right) d t .
\end{aligned}
$$

The second term already appears for null geodesics. The first term is smaller than the second one, since $w \simeq-1$ for these models. Therefore the conclusions for null geodesics are valid also for timelike geodesics close to these directional singularities.

In order to have finite integrals of (6) it is necessary that $\ddot{x}$ tends to zero when $t$ tends to $-\infty$, since $e^{-x}=a^{-1}$ tends to infinity for directional singularities, and hence $x$ tends to $-\infty$ either.

The function $x$ should be then a divergent function of time with decreasing concavity $\ddot{x}$, asymptotically tending to zero. This happens with functions which behave asymptotically as $x(t) \simeq-(-t)^{p}$, with $0<p<2$. Faster diverging functions have nonzero asymptotic acceleration and functions decreasing more slowly do not diverge at infinity.

These sort of functions produce divergent integrals of the Ricci curvature and hence we are to conclude that all Type $\infty$ singularities are strong according to Tipler's and Królak's criteria. 


\section{THE MODEL}

The model proposed in [14] has a scale factor of the form

$$
a(t)=\left\{\begin{array}{ccc}
a_{E} e^{-\frac{1}{6 \gamma}\left(1+\frac{2 H_{f}}{H_{e}}+\sqrt{\frac{8 H_{f}}{H_{e}}}\right)\left[e^{-3 \gamma H_{e} t}-1\right]} e^{\frac{H_{e}}{2} t} \quad \text { if } \quad t<0 \\
a_{E}\left(\frac{3 \gamma}{2}\left(H_{e}+\sqrt{2 H_{e} H_{f}}\right) t+1\right)^{\frac{2}{3 \gamma}} e^{H_{f} t} \quad \text { if } \quad t \geq 0,
\end{array}\right.
$$

where $a_{E}, \gamma, H_{e}, H_{f}, H_{E}$ are parameters of the model.

Taking into account the values of these parameters in the model, the scale factor can be approximated as

$$
a(t) \simeq\left\{\begin{array}{ccc}
a_{E} e^{-\frac{1}{6 \gamma}\left[e^{-3 \gamma H_{e} t}-1\right]} e^{\frac{H_{e}}{2} t} & \text { for } & t<0 \\
a_{E}\left(\frac{3 \gamma}{2} H_{e} t+1\right)^{\frac{2}{3 \gamma}} e^{H_{f} t} & \text { for } & t \geq 0 .
\end{array}\right.
$$

We are interested in the behavior of the model for very small negative $t$. For that era, the barotropic index of the model is

$$
w(t) \simeq-1+\frac{2}{18 \gamma} e^{3 \gamma H_{e} t}, \quad h(t) \simeq \frac{e^{3 \gamma H_{e} t}}{6 \gamma} .
$$

In [14] it is shown that this model has no big bang singularity and there is no initial singularity in cosmic time. However, a singularity appears at finite proper time in the past for noncomoving observers.

This can be derived within our formalism for this model and similar ones, since in this case it is clear that $h(t)$ is an integrable function of coordinate time and therefore the model has a Type $\infty$ singularity of the first kind in our classification $\left(a_{\infty}=0, \rho_{\infty}=\infty, p_{\infty}=-\infty, w_{\infty}=-1\right)$.

\section{CONCLUDING REMARKS}

We have shown that the model in [14] and similar inflationary models [15] with the property

$$
\int_{-\infty}^{T} a(t) d t<\infty
$$

for some time $T$ have a directional singularity as initial singularity, which is accessible in finite proper time only for null geodesics and timelike geodesics with finite linear momentum $P$. Comoving observers, following cosmological fluid worldlines, have not experienced the initial singularity, since it would have taken them infinite proper time to reach present time. Their geodesic trajectories are complete toward the past.

This does not happen in other cosmological models for which there is no such discrepancy between the finiteness of proper time and coordinate time lapses.

The absence of a big bang singularity is an interesting feature for a cosmological model, even though the curvature still blows up at the new singularity. Milder singularities with vanishing, instead of diverging, energy density and pressure could be obtained with similar models, but with nonintegrable $h(t)$.

For a model starting with a big bang singularity, the proper time of comoving observers is finite and defines the maximum age of the universe that can be experienced by nonaccelerated observers.

On the contrary, for a model with a little bang singularity, the age of the universe in the previous sense is infinite and the proper time as measured by nonaccelerated observers can be as large as desired by diminishing their linear momentum $P$.

It is an intriguing feature the idea of initial singularity in these models, with observers for which the universe extends indefinitely to the past, avoiding the singularity. However, as it has been pointed in Sec. II, this is a pure classical analysis. It is expected that the necessary quantum effects to be considered on approaching the singularities may appease them as it has happened in other instances.
[1] A. G. Riess et al. (Supernova Search Team Collaboration), Astron. J. 116, 1009 (1998); S. Perlmutter et al. (Supernova Cosmology Project Collaboration), Astrophys. J. 517, 565 (1999).

[2] T. M. Davis et al., Astrophys. J. 666, 716 (2007).

[3] W. M. Wood-Vasey et al. (ESSENCE Collaboration), Astrophys. J. 666, 694 (2007).

[4] B. Leibundgut, in Reviews of Modern Astronomy, edited by R. E. Schielicke (Wiley-VCH, New York, 2004), vol. 17.

[5] D. N. Spergel et al. (WMAP Collaboration), Astrophys. J. Suppl. Ser. 148, 175 (2003); 170, 377 (2007); J. Dunkley et al. (WMAP Collaboration), Astrophys. J. Suppl. Ser. 180, 306 (2009); E. Komatsu et al. (WMAP Collaboration), Astrophys. J. Suppl. Ser. 180, 330 (2009).

[6] T. Padmanabhan, AIP Conf. Proc. 861, 179 (2006).

[7] A. Albrecht et al., arXiv:astro-ph/0609591.

[8] V. Sahni and A. Starobinsky, Int. J. Mod. Phys. D 15, 2105 (2006).

[9] R. Maartens, J. Phys. Conf. Ser. 68, 012046 (2007).

[10] R. Durrer and R. Maartens, Gen. Relativ. Gravit. 40, 301 (2008).

[11] T. Padmanabhan, Gen. Relativ. Gravit. 40, 529 (2008). 
[12] L. Fernández-Jambrina and R. Lazkoz, Phys. Lett. B 670, 254 (2009).

[13] J. D. Barrow and A. A. H. Graham, Phys. Rev. D 91, 083513 (2015).

[14] J. Haro, J. Amorós, and S. Pan, Phys. Rev. D 93, 084018 (2016).

[15] A. Borde, A. H. Guth, and A. Vilenkin, Phys. Rev. Lett. 90, 151301 (2003).

[16] L. Fernández-Jambrina, Phys. Lett. B 656, 9 (2007).

[17] L. Fernández-Jambrina, Phys. Rev. D 90, 064014 (2014).

[18] C. Cattoën and M. Visser, Classical Quantum Gravity 22, 4913 (2005).

[19] S. Nojiri, S. D. Odintsov, and S. Tsujikawa, Phys. Rev. D 71, 063004 (2005)

[20] M. P. Dąbrowski and K. Marosek, J. Cosmol. Astropart. Phys. 02 (2013) 012.

[21] A. V. Yurov, Phys. Lett. B 689, 1 (2010).

[22] M. P. Dąbrowski, K. Marosek, and A. Balcerzak, Mem. Soc. Astron. Ital. 85, 44 (2014).

[23] P. H. Frampton, K. J. Ludwick, and R. J. Scherrer, Phys. Rev. D 84, 063003 (2011); P. H. Frampton, K. J. Ludwick, S. Nojiri, S. D. Odintsov, and R. J. Scherrer, Phys. Lett. B 708, 204 (2012).

[24] P. H. Frampton, K. J. Ludwick, and R. J. Scherrer, Phys. Rev. D 85, 083001 (2012).

[25] M. Bouhmadi-Lopez, A. Errahmani, P. Martin-Moruno, T. Ouali, and Y. Tavakoli, Int. J. Mod. Phys. D 24, 1550078 (2015).

[26] R. R. Caldwell, M. Kamionkowski, and N. N. Weinberg, Phys. Rev. Lett. 91, 071301 (2003).

[27] J. D. Barrow, Classical Quantum Gravity 21, L79 (2004); S. Nojiri and S. D. Odintsov, Phys. Lett. B 595, 1 (2004); J. D. Barrow, Classical Quantum Gravity 21, 5619 (2004); K. Lake, Classical Quantum Gravity 21, L129 (2004); S. Nojiri and S. D. Odintsov, Phys. Rev. D 70, 103522 (2004); M. P. Dąbrowski, Phys. Rev. D 71, 103505 (2005); L. P. Chimento and R. Lazkoz, Mod. Phys. Lett. A 19, 2479 (2004); M. P. Dąbrowski, Phys. Lett. B 625, 184 (2005); J. D. Barrow, A. B. Batista, J. C. Fabris, and S. Houndjo, Phys. Rev. D 78, 123508 (2008); J. D. Barrow and S. Z. W. Lip, Phys. Rev. D 80, 043518 (2009); S. Nojiri and S. D. Odintsov, Phys. Rev. D 78, 046006 (2008); J. D. Barrow, S. Cotsakis, and A. Tsokaros, Classical Quantum Gravity 27,
165017 (2010); J. D. Barrow, S. Cotsakis, and A. Tsokaros, arXiv:1003.1027; P. Singh, Phys. Rev. D 85, 104011 (2012); T. Denkiewicz, M. P. Dąbrowski, H. Ghodsi, and M. A. Hendry, Phys. Rev. D 85, 083527 (2012).

[28] J. D. Barrow, G. J. Galloway, and F. J. Tipler, Mon. Not. R. Astron. Soc. 223, 835 (1986).

[29] Y. Shtanov and V. Sahni, Classical Quantum Gravity 19, L101 (2002).

[30] V. Gorini, A. Y. Kamenshchik, U. Moschella, and V. Pasquier, Phys. Rev. D 69, 123512 (2004).

[31] A. O. Barvinsky, C. Deffayet, and A. Yu. Kamenshchik, J. Cosmol. Astropart. Phys. 05 (2010) 034.

[32] L. Fernández-Jambrina and R. Lazkoz, Phys. Rev. D 70, 121503 (2004).

[33] M. Bouhmadi-López, P. F. Gonzalez-Díaz, and P. MartínMoruno, Phys. Lett. B 659, 1 (2008).

[34] F. J. Tipler, Phys. Lett. 64A, 8 (1977)

[35] A. Królak, Classical Quantum Gravity 3, 267 (1986).

[36] L. Fernández-Jambrina and R. Lazkoz, Phys. Rev. D 74, 064030 (2006).

[37] J. D. Barrow and C. G. Tsagas, Classical Quantum Gravity 22, 1563 (2005)

[38] M. P. Dąbrowski and T. Denkiewicz, Phys. Rev. D 79, 063521 (2009).

[39] V. Sahni and Y. Shtanov, Phys. Rev. D 71, 084018 (2005).

[40] L. Fernández-Jambrina, Phys. Rev. D 82, 124004 (2010).

[41] S. W. Hawking and G. F. R. Ellis, The Large Scale Structure of Space-time (Cambridge University Press, Cambridge, England, 1973).

[42] M. V. Fischetti, J. B. Hartle, and B. L. Hu, Phys. Rev. D 20 , 1757 (1979); A. A. Starobinsky, Phys. Lett. 91B, 99 (1980); T. Azuma and S. Wada, Prog. Theor. Phys. 75, 845 (1986); J. Haro, J. Amorós, and E. Elizalde, Phys. Rev. D 83, 123528 (2011); E. D. Carlson, P. R. Anderson, J. R. Einhorn, B. Hicks, and A. J. Lundeen, arXiv:1607.01699.

[43] P. Singh, Classical Quantum Gravity 26, 125005 (2009); A. Corichi and P. Singh, Phys. Rev. D 80, 044024 (2009); P. Singh and F. Vidotto, Phys. Rev. D 83, 064027 (2011); K. Bamba, J. Haro, and S. D. Odintsov, J. Cosmol. Astropart. Phys. 02 (2013) 008.

[44] G. F. R. Ellis and B. G. Schmidt, Gen. Relativ. Gravit. 8, 915 (1977).

[45] C. J. S. Clarke and A. Królak, J. Geom. Phys. 2, 127 (1985). 